\title{
Research and Application of Rubber Tree Quantitative Fertilization System Control Based on PLC
}

\author{
Yeqin Wang ${ }^{1}$, Yuan Zhang ${ }^{1 *}$, Yiguo Deng ${ }^{1 *}$, Lijiao Wei ${ }^{1}$,and Shengli Liu ${ }^{1}$ \\ ${ }^{1}$ Institute of Agricultural Machinery, Chinese Academy of Tropical Agricultural Sciences, China
}

\begin{abstract}
In this paper, the quantitative control method and system control process of the natural rubber forest quantitative fertilization system are introduced on the basis of the demand for the quantitative fertilization of natural rubber forest and the application process of the rubber forest fertilizing machine. The selection of PLC, frequency converter type selection and software design process are described in detail.
\end{abstract}

\section{Introduction}

In recent years, with the development of Internet technologies, computer technologies, and modern electronic information technologies, our country's agriculture has slowly been produced from traditional agricultural methods to precision agriculture.Quantitative fertilization is an important part of precision agriculture and has achieved significant economic and social benefits abroad. The research on quantitative implementation technology of rubber forest in China started relatively late. The development of fertilization control system for application of granular fertilizer or powdered fertilizer in China is not yet mature, and the degree of automation is relatively low. Therefore, according to the practical needs of fertilizing the trenches for rubber trees, a quantitative fertilizer application system for rubber fields was developed based on the existing fertilizer applicators.

\section{Composition of quantitative fertilization system}

Quantitative fertilization system hardware is to select the fertilizer with the disk rotation, row fertilizer baffle-type fertilizer, through the use of motor driven worm gear reducer to the power to the fertilizer turntable to achieve fertilization ${ }^{[1]}$. Quantitative fertiliser machine row fertilizer structure is shown in Figure 1, from the fertilizer barrel, fertilizer turntable, row fat hopper, row fertilizer baffle, worm gear reducer, motor, level sensor, speed sensor, fertilizer pipe, weighing sensor, etc. composition.

For quantitative fertilization control, the flow rate of the fertilizer is automatically calculated and the speed of the motor is adjusted according to the input weight and time of the row of fertilizer to achieve the set flow rate requirement. The fertilizing structure of the quantitative fertilization system is shown in Figure 1. Quantitative fertilization system gives the total weight and time of fertilization and power up, the motor starts to rotate, the microprocessor controls the motor speed according to the current operation; the motor rotates and transmits the power to the worm gear reducer worm gear; the reducer transmits power to the flange Fertilizer turntable. During the rotation of the fertilizer turntable, the fertilizer in the fertilizer tank is guided to the edge of the fertilizer turntable by the action of its own gravity; Fertilizer at the

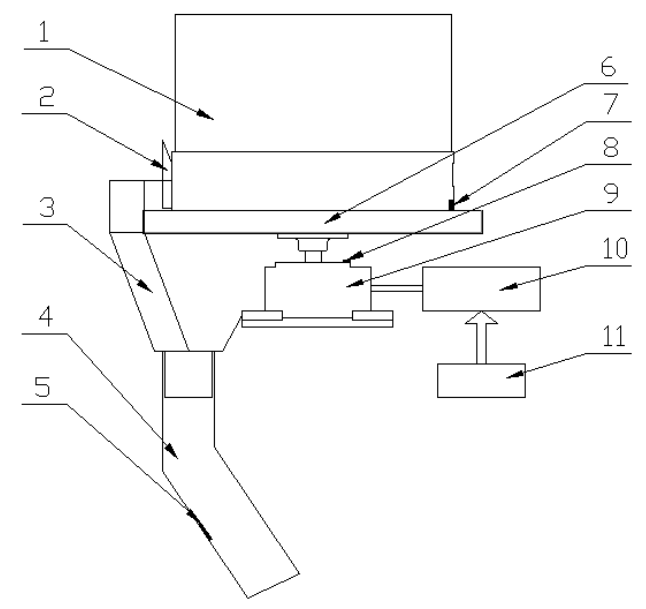

1. Fertilizer barrel; 2. Composting baffle; 3 . Composting hopper; 4. Row fertilizer tube; 5. Load cell 6. Fertilizer turntable; 7. Level sensor 8. Speed sensor; 9. Worm gear reducer 10. Motor; 11. Inverter

Figure 1. Fertilizer system structure schematic

edge of the fertilizer turntable is scraped by a row of fatretaining baffles into a row of fat hoppers; a load cell is weighed through a fat-feeding tube; a load cell outputs a voltage signal according to the magnitude of the applied force, and is amplified by the transmitter to output a measurement level signal proportional to the flow rate of the fertilizer.The signal is sent to the interface of the

*Corresponding author: Yeqin Wang: 441213852@qq.com 
upper computer. After sampling and converted into a flow signal, the current flow value is displayed on the upper computer. At the same time, the flow signal is sent to the PLC interface and compared with the ratio of the total fertilization weight and time set by the upper computer, and then the adjustment operation is performed. The control amount is sent to the inverter to change the output value of the inverter. Change the speed of the drive motor. Adjust the given amount and make it equal to the set value to complete the quantitative fertilization process.

Flow is the weight of fertilizer that passes through the fertilizer pipe at a certain time. The weighing sensor is the instantaneous flow, the upper computer gives the ratio of the average flow rate, that is, the ratio of the total weight to the time, and the two are deviated in the realtime measurement. In the actual control of the flow, the most widely used PID regulation is used in the industrial control. According to the flow deviation, the control quantity is controlled by the proportion, the integral and the differential calculation. The relation between the input and output (error) of the control quantity is expressed as follows in the time domain.

$$
u(t)=k\left[e(t)+\frac{1}{T} \int_{0}^{t} e(t) d(t)+\frac{H d e(t)}{d t}\right]
$$

In the formula, e(t) denotes the error that is the controller input; $\mathrm{u}(\mathrm{t})$ is the controller output; $\mathrm{k}$ is the proportional coefficient; $\mathrm{T}$ is the integral time constant; $\mathrm{H}$ is the derivative time constant. Figure 2 shows the system flow PID closed-loop regulation structure. When the fertilization process is automatically adjusted, according to the fertilization requirements, the total amount of fertilizer and the time to adjust the fertilization flow are set by the upper computer. Flow measurement control is a combination of measurement deviation and frequency control. It has the advantages of simple structure, good stability, reliable operation and easy adjustment.

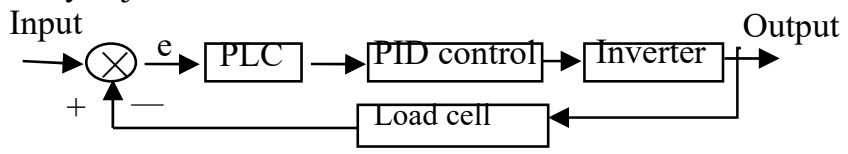

Figure 2. Flow PID closed-loop regulation

\section{System control process}

When the system begins to work, first step of the system program is initialized, and the total amount and time of input fertilization are set through the upper computer or the touch screen to check whether the fertilizer barrel has fertilizer material. If there is no fertilizer, fertilizer is sent to the fertilizer barrel, the quantitative fertilizer system is started, the weighing sensor is weighed and measured in real time, and the CPU calculates the real-time flow and the accumulated flow. If there is a deviation between the set flow and the actual flow, the regulator compares the deviation of the set value from the actual flow according to the system control requirements. The PID adjustment changes the output signal to control the speed adjustment of the inverter to the DC motor, thereby realizing constant flow control ${ }^{[2]}$. The main program control flow of the system is shown in Figure 3.

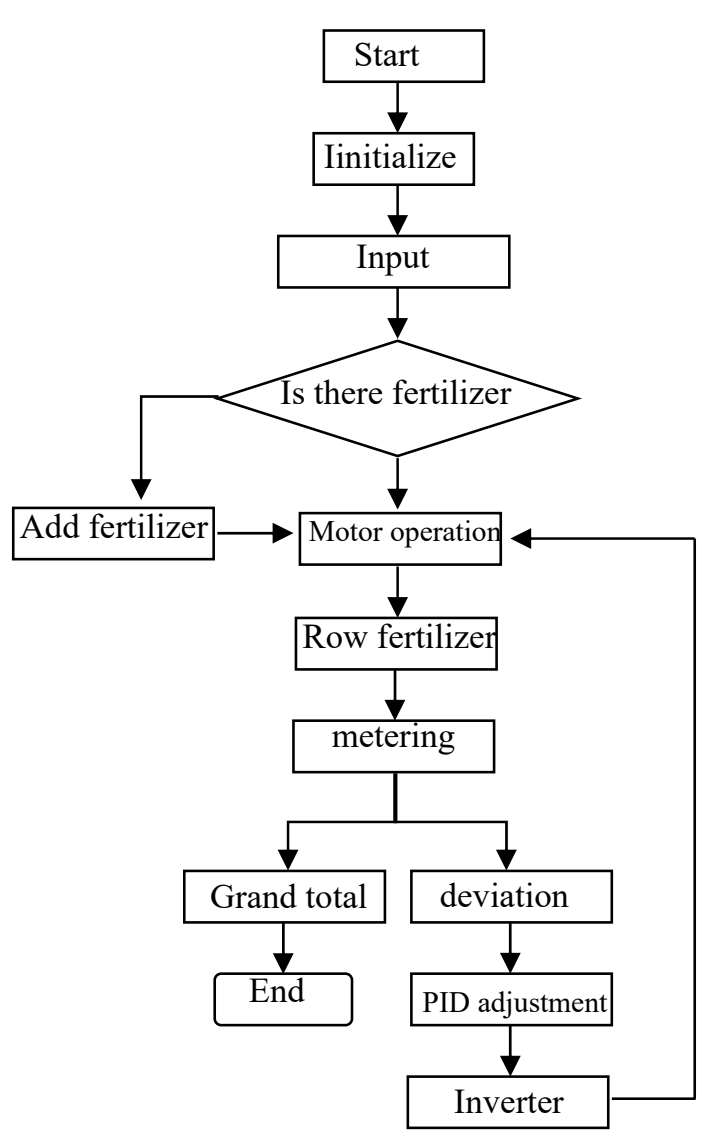

Figure 3. Control flow chart

\section{PLC control system hardware settings}

Quantitative fertilization in the system is controlled by the PLC and the upper computer. The system consists of a speed sensor, a load cell, and a frequency converter that form the controlled object. The start and stop of the motor is controlled by the switch quantity. The PLC digital output signal is used as the input signal of the control terminal of the frequency converter. After the frequency converter modulates and outputs the high frequency pulse to the motor, the speed sensor measures the speed of the motor. The system requires 3 digital input signals, 5 digital input signals and 5 digital output signals. The total number of $\mathrm{I} / \mathrm{O}$ points is 13 .

\subsection{PLC selection Inverter selection}


Since the number of $\mathrm{I} / \mathrm{O}$ points of the system controlled object does not exceed 16 and the system process requirements, scanning speed, self-diagnosis function, etc., Mitsubishi FX2N-48 is selected. In addition to meeting the design requirements, the selected PLC has a certain margin ${ }^{[3]}$. Mitsubishi FX series can be connected to the expansion module in the basic unit, can be a flexible combination of 16-256 points input and output. It can be choosed $16 / 32 / 48 / 64 / 80 / 128$-point host; can be used the minimum expansion module 8 points to expand, it can freely be choosed according to the power supply and output form ${ }^{[4]}$. Program capacity built-in 8k step can be expanded to $16 \mathrm{k}$ steps.

\subsection{Inverter selection}

The frequency converter is the hardware of the actuator of the quantitative fertilization system. It changes the frequency of the motor by changing its frequency, and thus changed the rotational speed of the row of fertilizer. The choice of frequency converter must be selected based on the power and current of the motor. According to the rated power of the drive motor of the fertilizer applicator $2 \mathrm{KW}$, rated voltage $380 \mathrm{~V}$, speed $720 \mathrm{r} / \mathrm{min}$, FR-A540 series inverter, select the speed range $120 \sim 1200 \mathrm{r} / \mathrm{min}$. According to quantitative fertilization production process requirements for PID control[5].

\section{Software design of PLC control system}

This system control mainly includes PLC control program, PC control program and communication program. PLC control program as a lower computer control, the entire control system data acquisition and processing has an important role. It accepts external switch signals (buttons, relay contacts), collects speed data and weight data to determine the current system status and output signals to control contactors, relays and other devices to complete the corresponding control tasks. In addition, another important task is to accept the control commands of the PC (upper computer) for automatic quantitative control. The Mitsubishi PLC control program is divided into a main program and 6 subroutines, and the proportional integral derivative control program in the subroutine is the core of the quantitative fertilization system. The proportional integral derivative control command PID is used for analog closed-loop control, [S1•][S2•] each use a data register, $[\mathrm{S} 1 \cdot]$ is used to store the set target value, [S2-] is used to set the current value to be measured, $[\mathrm{S} 3 \cdot]$ is the first address defined by the user for the PID instruction. The range is D0 to D7975, and 25 continuous data registers starting from [S3.] are required, where $[\mathrm{S} 3 \cdot]^{\sim}[\mathrm{S} 3 \cdot]+6$ Set the control parameters ${ }^{[6]}$. $\left[D^{\cdot}\right]$ uses a separate data register to store the output value. When the program is executed, the result of the operation is stored in $\left[\mathrm{D}^{\cdot}\right]$. The function of PID instruction is to calculate the adjustment value according to PID algorithm after receiving one input data.

\section{Conclusion}

The system adopts the method of PLC control and PID adjustment to design a quantitative fertilization system. Through logical judgment, the speed of the variable worm gear reducer is adjusted to achieve a quantitative effect of fertilization, and the intelligence level of fertilization operations and the accuracy of fertilization are improved.

\section{Acknowledgements}

This work was financially supported by the Natural Science Foundation of Hainan Provincial(518QN279); Central Public-interest Scientific Institution Basal Research Fund for Chinese Academy of Tropical Agricultural Sciences(1630132017003);Public Welfare Research and Capacity Building of Guangdong Province (2017A02028012);2017 provincial agricultural development and rural work special "natural rubber processing of key technologies in Thailand application"(Yue Nongji [2017] No.39)and Collaborative innovation and platform environment construction special of Guangdong Province (2017A040406003).

I would like to thank the members of the research group for their selfless help, especially the communication author Zhang Yuan and Deng Yiguo,and the guangba farms in Hainan Province for the project test of strong support.

\section{REFERENCES}

1. Qin Chaomin, Liu Junhui. Study Design of Manure Distribution Parts for Centrifugal Manure Spreader[J]. Journal of Agricultural Mechanization Research, 2006,(10):100-102.

2. Sun Xiaoquan, Qian Shaoming. Design and Implementation of PID Controller Based on PLC[J]. Applied Science and Technology, 2008, 35(6):29-32 
3. Wang Guohai, programmable controller and its application $[\mathrm{M}]$. Beijing: China Labor Social Security Press, 2007:204

4. Yan Yingfu. PLC Practical Guide [M] Beijing: Beijing Normal University Press, 2011: 167-168

5. MITSUBISHI Mitsubishi FR-A540 Series Inverter Manual

6. Mitsubishi Automation Engineering Co., Ltd. Programmable Controller FX2N User's Manual [M]. Shanghai: Mitsubishi Automation Engineering Corporation, 2005. 